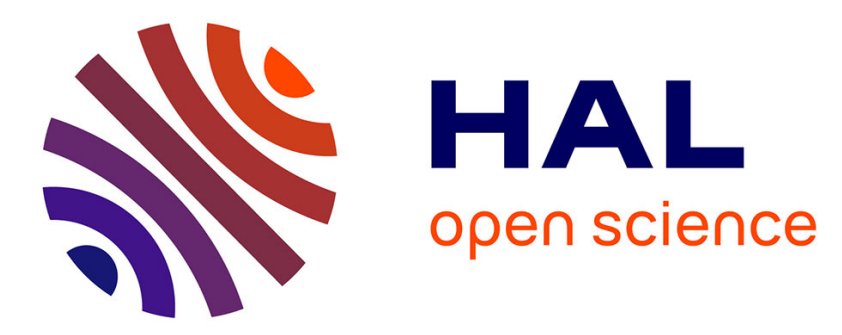

\title{
10GHz Demonstration of Four-Wave-Mixing in Photonic Crystal Waveguides
}

\author{
Kevin Lenglé, Akram Akrout, Marcia Costa E Silva, Laurent Bramerie, \\ Sylvain Combrié, Pierre Colman, Jean-Claude Simon, Alfredo de Rossi
}

\section{- To cite this version:}

Kevin Lenglé, Akram Akrout, Marcia Costa E Silva, Laurent Bramerie, Sylvain Combrié, et al. $10 \mathrm{GHz}$ Demonstration of Four-Wave-Mixing in Photonic Crystal Waveguides. 36th European Conference on Optical Communication (ECOC 2010), Sep 2010, Torino, Italy. pp.P2.24, 10.1109/ECOC.2010.5621546 . hal-00691527

\section{HAL Id: hal-00691527 \\ https://hal.science/hal-00691527}

Submitted on 26 Apr 2012

HAL is a multi-disciplinary open access archive for the deposit and dissemination of scientific research documents, whether they are published or not. The documents may come from teaching and research institutions in France or abroad, or from public or private research centers.
L'archive ouverte pluridisciplinaire HAL, est destinée au dépôt et à la diffusion de documents scientifiques de niveau recherche, publiés ou non, émanant des établissements d'enseignement et de recherche français ou étrangers, des laboratoires publics ou privés. 


\title{
10GHz Demonstration of Four-Wave-Mixing in Photonic Crystal Waveguides
}

\author{
Kevin Lengle ${ }^{(1)}$, Akram Akrout $^{(1)}$, Marcia Costa e Silva ${ }^{(1)}$, Laurent Bramerie ${ }^{(1)}$ \\ Sylvain Combrié(2) Pierre Colman $^{(2)}$, Jean-Claude Simon ${ }^{(1)}$ and Alfredo De Rossi ${ }^{(2)}$ \\ (1) Université Européenne de Bretagne (UEB), CNRS-Foton laboratory (UMR 6082)/Université de Rennes 1, Enssat, \\ (2) Thales Research and Technology, 1, Av. A. Fresnel, 91767 Palaiseau, France,
} BP 80518, 22305 Lannion Cedex, France,

$\bowtie$ alfredo.derossi@thalesgroup.com

\begin{abstract}
We report time resolved measurement of the nonlinear frequency conversion of a high repetition rate (10 $\mathrm{GHz}$ ) train of short pulses (up to $4 \mathrm{ps}$ ) in a $1.5 \mathrm{~mm}$ long Photonic Crystal waveguides. We provide direct evidence of fast response through time-resolved optical measurements. The conversion gain of about -40dB is achieved with about $60 \mathrm{~mW}$ of average power at the waveguide input.
\end{abstract}

\section{Introduction}

Four-wave-mixing (FWM) is an elegant technique to achieve a key functionality for ultra-fast optical processing. FWM is well-mastered in optical fibers ${ }^{1}$ and in semiconductor optical amplifiers ${ }^{5}$ The emergence of integrated photonics, with is potential for implementation of optical functions directly at the level of a computer architecture and for rapid growth of the aggregate bandwidth per single device, owing to massive parallelization, calls for solutions for efficient implementation of FWM at the level of a monolithic optical device. Moreover, miniaturization brings the opportunity for enhanced nonlinear effect with the promise of a decrease of the power levels required. In this respect, silicon nanowires have pioneered the field ${ }^{3}$. However, silicon suffers from strong nonlinear absorption and related free-carrier absorption, which limits the potential benefits. Therefore other materials and effects suitable for frequency conversion have been investigated, for instance parametric conversion in Periodically Poled Lithium Niobate (PPNL) waveguides ${ }^{10}$. The footprint here is set by the normalized conversion efficiency in PPNL waveguides (typically $250 \% \mathrm{~cm}^{-1} W^{-1}$ ) thus implying device lengths in the 1 to $10 \mathrm{~cm}$ range. Also, doped glasses ${ }^{2}$ and chalcogenides fibers, waveguides and tapered fibers ${ }^{8}$, which have been used to demonstrate all-optical regeneration, pulse compression and frequency conversion ${ }^{13}$. Another promising approach exploits the very large nonlinearity related to intersubband transitions in Antimonide-based multi-quantumwells ${ }^{4}$.

\section{Device description}

Here, we focus on Photonic Crystal waveguides. PhC waveguides are obtained by omitting a line in a 2D lattice of holes. The effective cross section of the optical mode is very small $\left(A_{e f f} \approx 0.5 \mu m^{2}\right)$. Interestingly, the dispersion differs remarkably from that of a ridge waveguide, in particular group velocity smaller than $c / 10$ are routinely achieved ${ }^{9}$.Owing to the very strong confinement of light and to the relatively slow group velocity which is achievable, the nonlinear response of the material is greatly enhanced. That has been shown in silicon. PhC on TPA-free materials, such as chalcogenides, have demonstrated their potential for FWM while $A l_{0.18} G a .82 A s\left(E_{g} \approx 1.65 \mathrm{eV}\right)$ has been used to demonstrate extremely high SPM gain in $\mathrm{PhC}$ waveguides $^{7}$. Both device footprint and power budget are one order of magnitude better than any other technology as a result of field enhancement due to the combined effect of confinement and reduced group velocity of the waveguide mode. We have developed highly nonlinear $\mathrm{PhC}$ based on III-V ${ }^{11}$ with very low insertion losses ${ }^{12}$. With that technology, highly efficient FWM has been demonstrated very recently ${ }^{14}$. One of the most striking properties of FWM based on pure Kerr effect, which is our case, is the potential for ultra fast response, which is here only limited by the dispersion of the waveguide and, ultimately, its transmission bandwidth. The sample used for the experiment is a self-standing membrane of InGaP, which is also TPA-free $\left(E_{g} \approx 1.9 \mathrm{eV}\right)$. The self phase modulation coefficient $\gamma$ ranges between $0.8 \times 10^{3} \mathrm{~W}^{-1} \mathrm{~m}^{-1}$ and $\approx 2 \times 10^{3} \mathrm{~W}^{-1} \mathrm{~m}^{-1}$, depending on the wavelength. However, stronger linear loss and dispersion in the slow light regime limit the benefit of higher nonlinearity. A mode adapting structure allows to optimize optical losses coupling (fig. 1).

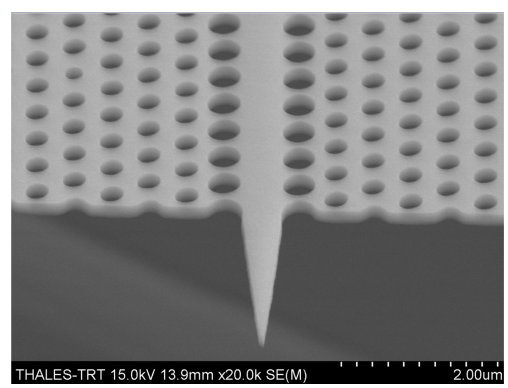

Fig. 1: Photonic Crystal waveguide picture

In the following section, we show for the first time the ultra-fast frequency conversion of high repetition rate (10 Ghz) train of short pulses (up to $4 \mathrm{ps}$ ) PhC waveguides using time-resolved techniques.

\section{Experiment setup}

Fig. 2 shows the experimental setup of FWM characterization. The pump signal is a mode-locked diode laser generating $1.5 \mathrm{ps}$ pulses with a time-bandwidth-product (TBP) of 0.35 at $10 \mathrm{GHz}$ repetition rate. After amplification and filtering, a variable pulse width between 4 
and 16 ps is obtained when using bandwith optical filter of 3 and $0.25 \mathrm{~nm}$ respectivelly . That signal is combined with a continuous wave tuneable laser providing the probe. Then we use a bandwith optical filter of $5 \mathrm{~nm}$ in order to filter out the spontaneous emission of the EDFAs for FWM signal viewing. Finally, the signals are

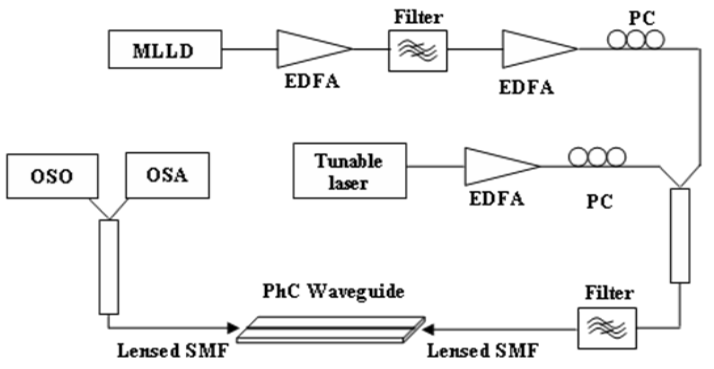

Fig. 2: Experimental setup for FWM characterization. EDFA is an Erbium doped fiber amplifier, $\mathrm{PC}$ is a polarization controller, OSA is an Optical spectrum analyzer, and OSO is an Optical sampling oscilloscope.

fed to the PhC waveguide with a fiber tapered tip providing a focused spot of $2 \mu \mathrm{m}$ diameter at $1 / e^{2}$ intensity. The transmitted light is collected using a second lensed fiber and measured using an optical spectrum analyzer (OSA). The total insertion losses are about $12 \mathrm{~dB}$ (e.g. fiber to waveguide to fiber efficiency is $5,6 \%$ ). Fig. 3 shows the linear transmission of the Photonic Crystal. The transmission of the sample is shown in figure 4 . Fig. 4 shows the typical spectra collected out of the waveguide in a FWM experiment. FWM idler and CW probe appear as symmetrical pairs as the detuning is changed. THe FWM signal disappears as the probe is off (black curve). Cross Phase modulation (XPM) is also induced by the pump on the CW probe.

\section{Results}

The FWM efficiency is defined as the FWM idler peak power collected at the output fiber divided the probe power at the input, namely $\eta_{F W M}=$ $P_{F W M}($ out, peak)/Probe $($ in $)$. This quantity is measured by the OSA and corrected with the duty cycle (namely $100 \mathrm{ps} / 16 \mathrm{ps}$, for $16 \mathrm{ps}$ pulses), as the OSA measures the average FWM signal. We stress that this is an external efficiency, e.g. fiber to fiber, as it already takes into account insertion losses. The result is shown

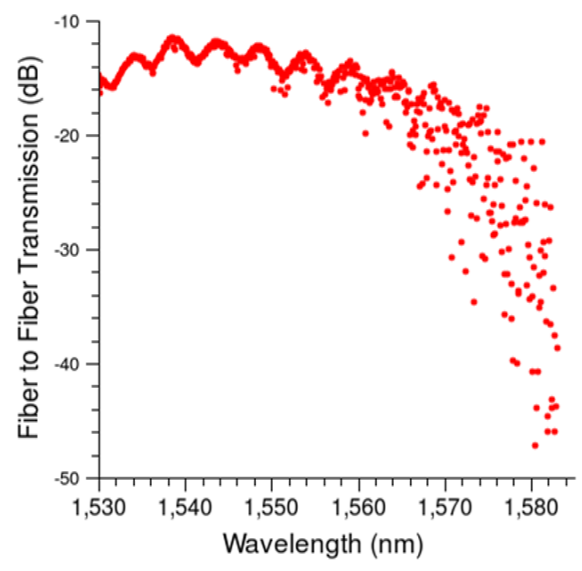

Fig. 3: Linear transmission of the $\mathrm{PhC}$ waveguide.

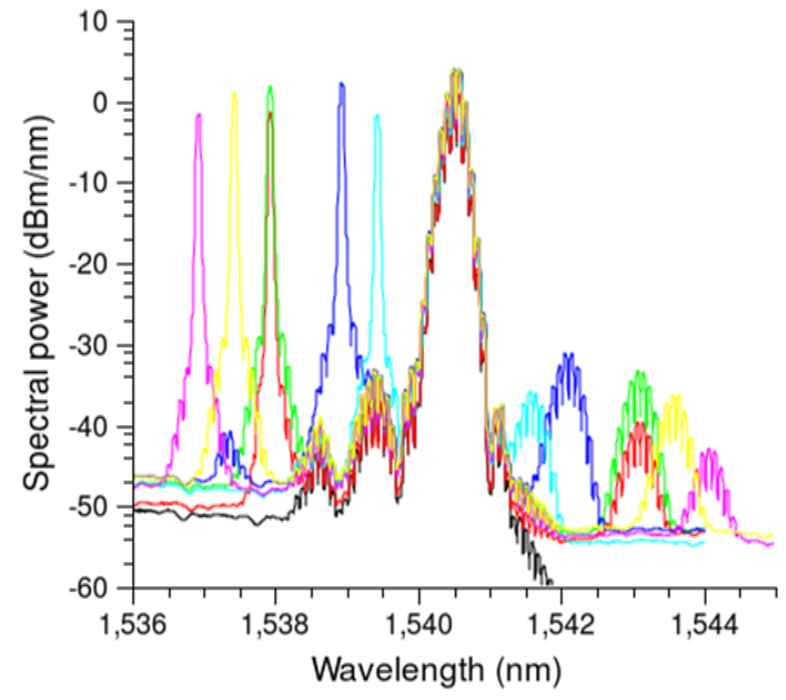

Fig. 4: Output spectra with CW signal, 4 ps pump and FWM idler. Colors distinguish different values of the probe-pump detuning. Black curve is without CW signal.

in fig.5. First, we measured the dependence on the detuning when the input power levels are $18 \mathrm{dBm}$ (pump, average) and $13 \mathrm{dBm}$ (probe) respectively. The FWM bandwidth is set by the dispersion and is here about $5 \mathrm{~nm}$, which is consistent with previous measurements (accounting for a difference in the dispersion due to a different operating wavelength). We also measure the same efficiency as a function of the pump power and we found a change of $15 \mathrm{~dB}$ of the FWM idler as the pump is changed from 12 to $20 \mathrm{dBm}$. We then investi- (a)

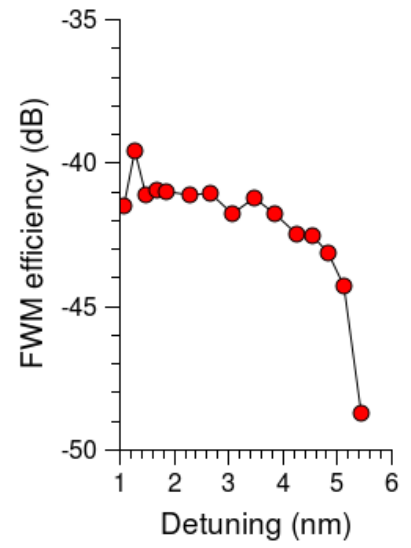

(b)

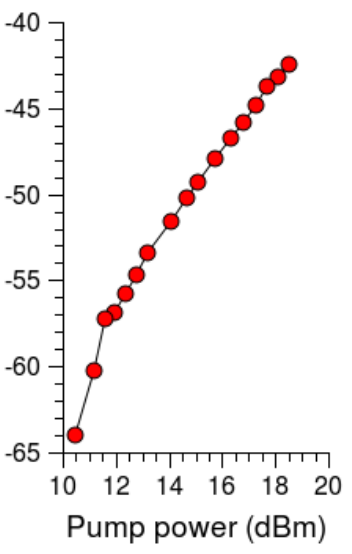

Fig. 5: FWM efficiency for 16 ps long input pulses. (a) efficiency vs. the detuning, pump (average) and probe power level are $18 \mathrm{dBm}$ and $13 \mathrm{dBm}$ respectively. (b) efficiency versus the pump power with detuning $=2 \mathrm{~nm}$.

gated the FWM signal using an optical sampling oscilloscope ("Picosolve") with a time resolution of 1 ps. We have investigated the time response under various conditions (pump power and pulse duration, detuning, ...). Here, we show results for experiments with 4 ps pulses. A filter is placed at the output fiber and centered on the FWM signal in order to filter pump and probe out. First, we show that when pump is on and the probe is off, the FWM signal is zero (red trace), consistent with fig.4. This supports the claim that the pump is effectively fil- 
tered out and does not reach the optical sampling oscilloscope. As both pump and probe are on, the FWM signal appears. The inset shows a close up of the pump and FWM temporal traces, demonstrating the ultra-fast response of the FWM effect in our waveguides, thereby ruling out the possibility of longer recovery times due to carrier dynamic. This is a very important point, confirming our previous findings ${ }^{6 ?}$ on low-repetition rate experiments.

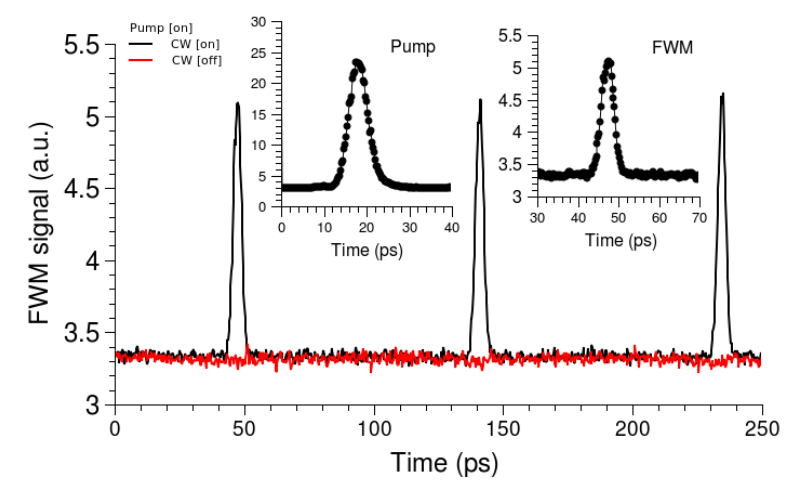

Fig. 6: Time-resolved traces using an optical sampling oscilloscope of the FWM signal generated by mixing a 4 ps pump and a CW probe (black). The red trace is with the probe set to off but still pump on and corresponds to the optical noise. Insets: left, the pump at the input, right, the FWM signal, with duration about 4 ps.

\section{Conclusion}

Owing to the very strong confinement of the optical field in PhCs and to the large nonlinear coefficients in III-V semiconductors, ultra-fast all-optical functions can be implemented in very small devices (length $\approx$ $1 \mathrm{~mm}$ ). Using a material which greatly minimizes nonlinear absorption, we were able to demonstrate ultrafast frequency conversion originating from the Kerr effect, with no noticeable effects related to carrier dynamics or thermal effect, despite the high repetition rate (10 $\mathrm{GHz}$ ). We believe that is an important step forward the use of Photonic Crystals for all optical processing.

\section{Acknowledgements}

This work was supported by the European project COPERNICUS.

\section{References}

1 E. Ciaramella and S. Trillo. All-optical signal reshaping via four-wave mixing in optical fibers. Photonics Technol. Lett., 12:849, 2000.

2 M. Ferrera, L. Razzari, D. Duchesne, R. Morandotti, Z. Yang, M. Liscidini, J. E. Sipe, S. Chu, B. E. Little, and D. J. Moss. Low-power continuous-wave nonlinear optics in doped silica glass integrated waveguide structures. Nat. Photon., 2:737, 2008.

3 H. Fukuda, K. Yamada, T. Shoji, M. Takahashi, T. Tsuchizawa, T. Watanabe, J. ichi Takahashi, and S. ichi Itabashi. Four-wave mixing in silicon wire waveguides. Opt. Express, 13(12):4629-4637, 2005.
4 K. A. S. G. T. M. T. H. G. W. Cong, R. Akimoto and $\mathrm{H}$. Ishikawa. Experimental and theoretical study of cross-phase modulation in ingaas/alassb coupled double quantum wells with a algaas coupling barrier. Phys. Rev. B, 80:035306, 2009.

5 A. B. H. Simos and D. Syvridis. Investigation of $a 2 r$ all-optical regenerator based on four-wave mixing in a semiconductor optical amplifier. J. Lightwave Technol., 22:595, 2004.

6 C. Husko, S. Combrié, Q. V. Tran, F. Raineri, C. W. Wong, and A. D. Rossi. Non-trivial scaling of selfphase modulation and three-photon absorptionin iii-v photonic crystal waveguides. Opt. Express, 17(25):22442-22451, 2009.

7 K. Inoue, H. Oda, N. Ikeda, and K. Asakawa. Enhanced third-order nonlinear effects in slowlight photonic crystal slab waveguides of linedefect. Opt. Expr., 17:7206, 2009.

8 E. C. Mägi, L. B. Fu, H. C. Nguyen, M. R. Lamont, D. I. Yeom, and B. J. Eggleton. Enhanced kerr nonlinearity in sub-wavelength diameter as2se3 chalcogenide fiber tapers. Opt. Express, 15(16):10324-10329, 2007.

9 M. Notomi. Phys. Rev. Lett., 2001.

10 T. Ohara, H. Takara, I. Shake, T. Yamada, M. Ishii, I. Ogawa, M. Okamoto, and S. Kawanishi. Highly stable $160-\mathrm{gb} / \mathrm{s}$ otdm technologies based on integrated mux/demux and drift-free pll-type clock recovery. IEEE Sel. Top. Q. Elect., 13:40, 2007.

11 C. H. Sylvain Combrĩ̃ (C) Quynh Vy Tran Alfredo De Rossi and P. Colman. High quality gainp nonlinear photonic crystals with minimized nonlinear absorption. Appl. Phys. Lett, 95:221108, 2009.

12 P. C. a. A. D. R. Quynh Vy Tran, Sylvain Combriã(C). Photonic crystal membrane waveguides with low insertion losses. Appl. Phys. Lett, 95:061105, 2009.

13 V. Ta'eed, N. J. Baker, L. Fu, K. Finsterbusch, M. R. E. Lamont, D. J. Moss, H. C. Nguyen, B. J. Eggleton, D.-Y. Choi, S. Madden, and B. LutherDavies. Ultrafast all-optical chalcogenide glass photonic circuits. Opt. Express, 15(15):9205-9221, 2007.

14 V. Eckhouse et al. Highly efficient four wave mixing in GalnP photonic crystal waveguides Opt. letters, to be published. 